\title{
A Fringe Field Switching Liquid Crystal Display With Fast Grayscale Response Time
}

\author{
Daming Xu, Haiwei Chen, Shin-Tson Wu, Fellow, IEEE, Ming-Chun Li, Seok-Lyul Lee, and Wen-Ching Tsai
}

\begin{abstract}
A fast-response fringe-field switching (FFS) liquid crystal display using patterned common electrodes is proposed. By applying a restoring pulse voltage on common electrodes, the relaxation process of liquid crystals is expedited by the electric field. The average gray-to-gray decay time is over sevenfold faster compared with the conventional FFS mode.
\end{abstract}

Index Terms-Fast response, fringe field switching (FFS), liquid crystal display (LCD).

\section{INTRODUCTION}

$\mathbf{F}$ RINGE field switching liquid crystal display (FFS LCD) [1]-[4] has been widely used in smartphones and tablets because it possesses several attractive features, such as wide view for multiple viewers, high resolution for Retina display, low power consumption for long battery life, pressure resistance for touch panels. Nevertheless, some technical barriers remain to be overcome, such as image sticking [5], [6], thin-film transistor (TFT) charging time for high resolution LCDs [4], and relatively slow response time. Image sticking can be reduced by optimizing device and material properties [7], [8], while slow charging problem can be resolved by using an oxide TFT with high mobility [9]. However, the slow LC response time, which causes motion blurs and deteriorated image quality [10], remains a critical issue.

For FFS LCDs, the rise and decay times between gray level transitions are governed by the LC material properties and cell gap as [11]

$$
\begin{aligned}
\tau_{\text {rise }} & =\tau_{o} /\left[\left(V / V_{\mathrm{th}}\right)^{2}-1\right] \\
\tau_{\text {decay }} & =\tau_{o} /\left|\left(V_{b} / V_{\mathrm{th}}\right)^{2}-1\right| \\
\tau_{o} & =\gamma_{1} d^{2} / K_{22} \pi^{2}
\end{aligned}
$$

where $V$ is the applied voltage to the final gray level, $V_{b}$ is the bias voltage of the initial gray level, $V_{\text {th }}$ is the threshold voltage, $\gamma_{1}$ is the rotational viscosity, and $K_{22}$ is the twist elastic constant. From (1), both rise time and decay time could be slow when the grayscale voltages are close, especially in the vicinity of threshold. Compared to the rise process, which

Manuscript received December 10, 2014; revised January 14, 2015; accepted January 27, 2015. Date of publication January 29, 2015; date of current version March 24, 2015.

D. Xu, H. Chen, and S.-T. Wu are with the College of Optics and Photonics, University of Central Florida, Orlando, FL 32816 USA (e-mail: swu@ucf.edu).

M.-C. Li, S.-L. Lee, and W.-C. Tsai are with AU Optronics Corporation, , Hsinchu 300, Taiwan.

Color versions of one or more of the figures are available online at http:// ieeexplore.ieee.org.

Digital Object Identifier 10.1109/JDT.2015.2398315
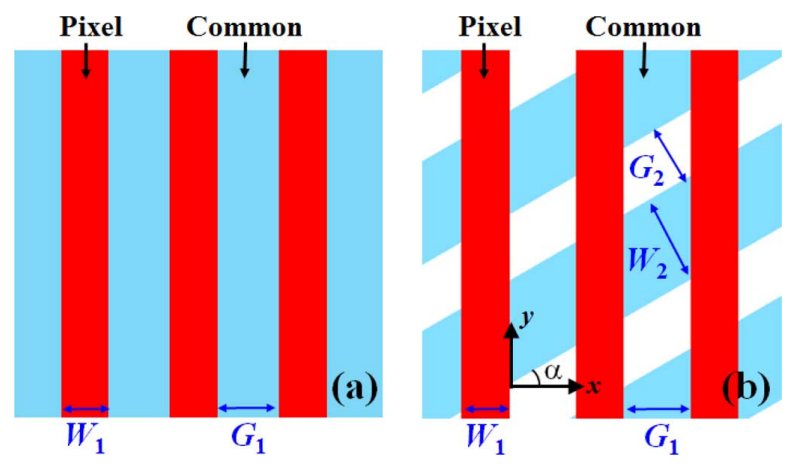

Fig. 1. Device structure of (a) FFS and (b) PCFFS cells.

is driven by an electric field, the decay process is usually slower since it is mainly governed by the restoring elastic force. Because of the small $K_{22}$, the decay time of a $3-\mu \mathrm{m}$ cell gap FFS LCD is usually $\sim 20 \mathrm{~ms}$ [12], which is too slow for TV applications. In contrast, the gray-to-gray (GTG) response time of a multi-domain vertical alignment (MVA) LCD TV is $\sim 5 \mathrm{~ms}$ [13], [14]. In order to reduce the response time of FFS mode, various approaches have been proposed, such as ultra-low viscosity LCs [12], double-side structures [15]-[17], reactive mesogen-aligned cells [18], [19], triode driving using a vertical field to expedite the decay process [20]-[22], etc. However, each approach has its own pros and cons. Hence, there is a need to develop a fast-switching FFS LCD without sacrificing its intrinsic advantages.

In this paper, we propose a new FFS mode using patterned common electrode, denoted as PCFFS, to achieve fast response time. The bright state and the dark state are achieved by applying fringe fields along different directions. With a restoring voltage pulse to expedite the decay process, we are able to achieve seven-fold faster GTG decay time than that of a conventional FFS LCD.

\section{Device Structure}

Fig. 1(a) and (b) illustrates the top view structure of FFS and PCFFS cells, respectively. In a conventional FFS cell, stripe-shaped pixel electrodes and planar common electrodes are formed on the bottom substrate separated by a passivation layer. However, in our PCFFS cell, the bottom common electrodes are stripe-shaped as well, setting at an angle $\alpha$ with respect to the $x$ axis. Same as FFS cells, for the purpose of achieving low driving voltage the homogeneous rubbing angle is set at $10^{\circ}$ and $80^{\circ}$ w.r.t. the $x$ axis for PCFFS cells using a negative (n-PCFFS) and positive (p-PCFFS) LC, respectively [2]. The cell gap is optimized at $\lambda=550 \mathrm{~nm}$ with $d \Delta n=$ 
TABLE I

Properties of LC Mixtures Studied $\left(\mathrm{T}=23^{\circ} \mathrm{C}\right.$ AND $\lambda=550 \mathrm{~nm}$ )

\begin{tabular}{|c|c|c|c|c|c|c|}
\hline $\mathrm{LC}$ & $\begin{array}{c}\gamma_{1} \\
(\mathrm{mPa} \cdot \mathrm{s})\end{array}$ & $\Delta \varepsilon$ & $\Delta n$ & $\begin{array}{c}K_{11} \\
(\mathrm{pN})\end{array}$ & $\begin{array}{c}K_{22} \\
(\mathrm{pN})\end{array}$ & $\begin{array}{c}K_{33} \\
(\mathrm{pN})\end{array}$ \\
\hline UCF-N2 & 94.7 & -3.8 & 0.117 & 15.8 & 7.2 & 14.0 \\
\hline DIC-LC3 & 62.0 & 9.0 & 0.111 & 10.9 & 5.6 & 13.8 \\
\hline
\end{tabular}

$360 \mathrm{~nm}$ for n-PCFFS and $380 \mathrm{~nm}$ for p-PCFFS to obtain high transmittance. The LC cell is further interposed between two crossed linear polarizers with bottom polarizer's transmission axis parallel to the $\mathrm{LC}$ rubbing direction.

The operation principle of PCFFS is described as follows. In the null-voltage state, the linearly polarized light keeps its polarization when traversing through the LC layer and is therefore blocked by the crossed analyzer, resulting in a dark state. During turn-on process, a driving voltage is applied to the pixel electrodes while common electrodes are grounded. Same as FFS mode, fringe fields with strong horizontal components are generated here to reorient the LC directors. Hence, the linearly polarized light experiences phase retardation and is transmitted by the crossed analyzer. During decay process, the voltage on pixel electrodes is released and the electrodes are kept floated while a restoring voltage pulse is applied between neighboring common electrodes. The electric potential of floated top pixel electrodes are spontaneously determined by the restoring voltage [23]. The in-plane field generated by this restoring voltage would exert a strong torque to pull the LC directors back to their initial rubbing direction in addition to the existing elastic restoring torque [24]-[27]. Hence, the decay process is accelerated and faster decay time is obtained.

\section{Simulation Results: VT Characteristics}

The device performance of the proposed PCFFS are studied and optimized by using commercial simulator TechWiz LCD (Sanayi, Korea) and the electro-optic properties are calculated by the extended $2 \times 2$ Jones matrix method. To make a fair comparison between FFS and PCFFS cells, we use the same device configurations: pixel electrode width $W_{1}=2 \mu \mathrm{m}$, electrode gap $G_{1}=3 \mu \mathrm{m}$, pretilt angle $2^{\circ}$. The passivation layer between the pixel and common electrodes is $\mathrm{Si}_{3} \mathrm{~N}_{4}$ whose thickness is $d_{\text {pas }}=150 \mathrm{~nm}$ and dielectric constant is 7.5 whereas the alignment layer is $80-\mathrm{nm}$ thick polyimide with a dielectric constant of 3.8. The physical properties of the positive and negative LC materials studied are listed in Table I. The negative LC material UCF-N2 was developed by our group [28], while the positive $\Delta \varepsilon$ LC DIC-LC3 is a commercial material from DIC Japan.

\section{A. Voltage-Dependent Transmittance Curve}

Fig. 2 shows the simulated voltage-dependent transmittance (VT) curves of FFS and PCFFS cells employing a positive or a negative $\Delta \varepsilon$ LC material. Here, the $\alpha$ angle is set at $10^{\circ}$ and $-20^{\circ}$ for $\mathrm{p}$ - and n-PCFFS cell, respectively. The values of $\alpha$ angle are optimized to achieve the fastest response time, as will be explained later. The dimension of bottom common electrodes is set at $W_{2}=4 \mu \mathrm{m}$ and $G_{2}=2 \mu \mathrm{m}$. We can see that both pand n-PCFFS cells have nearly identical VT curves to the corresponding FFS cells. This is attractive as the proposed PCFFS

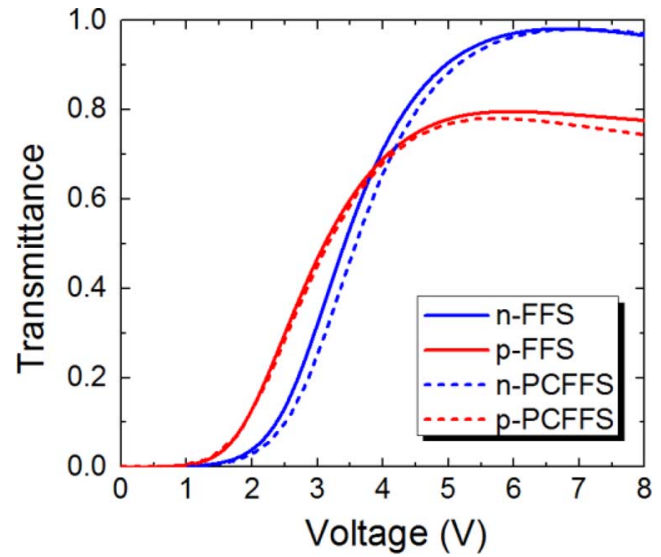

Fig. 2. Simulated VT curves of FFS and PCFFS modes using the positive and negative LCs listed in Table $1 . \lambda=550 \mathrm{~nm}$.
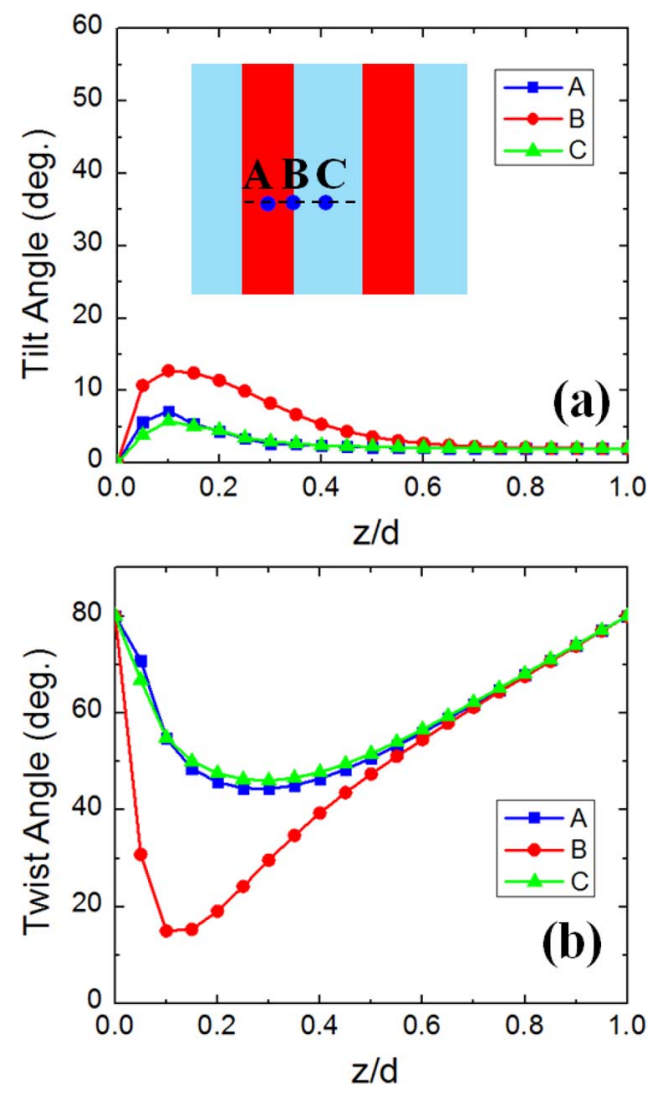

Fig. 3. Simulated (a) tilt angle and (b) twist angle distributions of the p-FFS cell at the full-bright state.

can achieve similar high transmittance as FFS does at the same voltage.

\section{B. LC Director Deformation Distribution}

To better understand the electro-optic properties of FFS and PCFFS modes, we investigate their LC distributions at the fullbright state. Due to space limitation, only the results of LC modes employing a positive $\Delta \varepsilon \mathrm{LC}$ are shown here; however, those using a negative LC exhibit the same trend.

Fig. 3(a) and 3(b) depicts the tilt and twist angles of LC directors at different positions [A to C as Fig. 3(a) shows] of a p-FFS cell. In order to obtain maximum phase retardation, the 

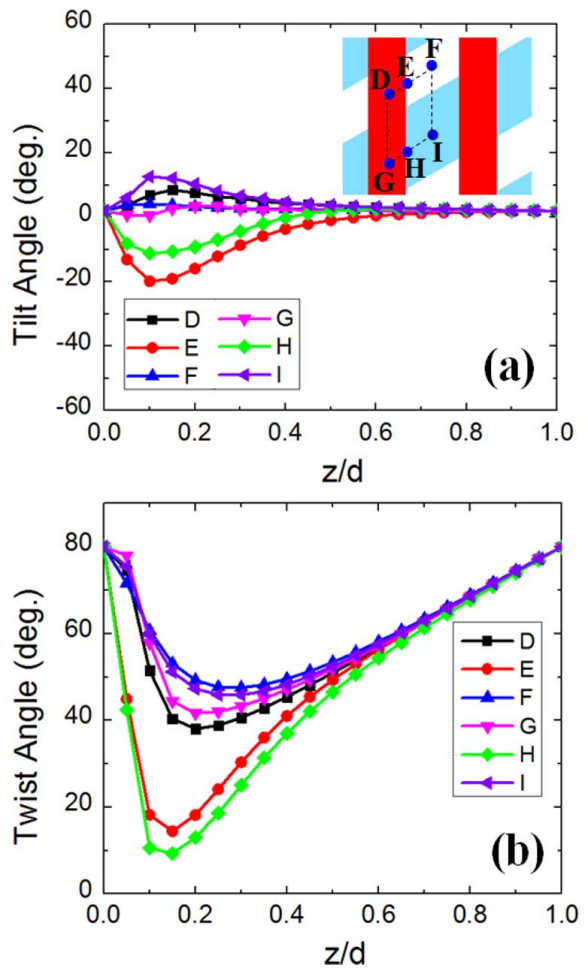

Fig. 4. Simulated (a) tilt angle and (b) twist angle distributions of the p-PCFFS cell at the full-bright state.

LC directors should be twisted at $45^{\circ}$ with respect to the rubbing direction, i.e., $35^{\circ}$ to the $\mathrm{x}$-axis. As plotted in Fig. 3(b), the LCs in the p-FFS cell are either over- or under-twisted, leading to an inefficient phase retardation at the center of electrodes and gaps. Meanwhile, the large tilt angle caused by the strong vertical field dramatically decreases the effective birefringence and hence the peak transmittance. This explains why p-FFS shows a lower transmittance than n-FFS mode [2].

In the single-domain p-FFS, the LC distributions are uniform along y axis; however, the patterned common electrodes in the PCFFS cell create multi-domain LC distribution. Figs. 4(a) and (b) depict the tilt and twist angles of LC directors at six different positions [D to I as Fig. 4(a) illustrates] in the p-PCFFS cell. As we can see, the LC tilt angle is less uniform along the $y$ direction in the p-PCFFS cell. The LC directors are tilted up by the electric field at positions D, F, G, and I, same as that in the p-FFS cell. In contrast, the tilt angle at some positions (E and $\mathrm{H})$ is negative, indicating the LC directors are tilted down at these positions. Nevertheless, the twist angles at all these positions are similar to the corresponding positions in the p-FFS cell. Please note the difference in tilt angles implies a multi-domain LC distribution is created in the p-PCFFS cell. This is because the patterned common electrodes create different electric field distributions; accordingly, the LC directors follow the electric fields and forms multi-domain structure. This feature helps to widen viewing angle, as will be outlined below.

\section{Viewing Angle}

To widen the viewing angle of single-domain FFS LCDs, compensation films are required. In terms of film configuration, a biaxial film or a pair of positive $\mathrm{A}$ and positive $\mathrm{C}$ uniaxial films

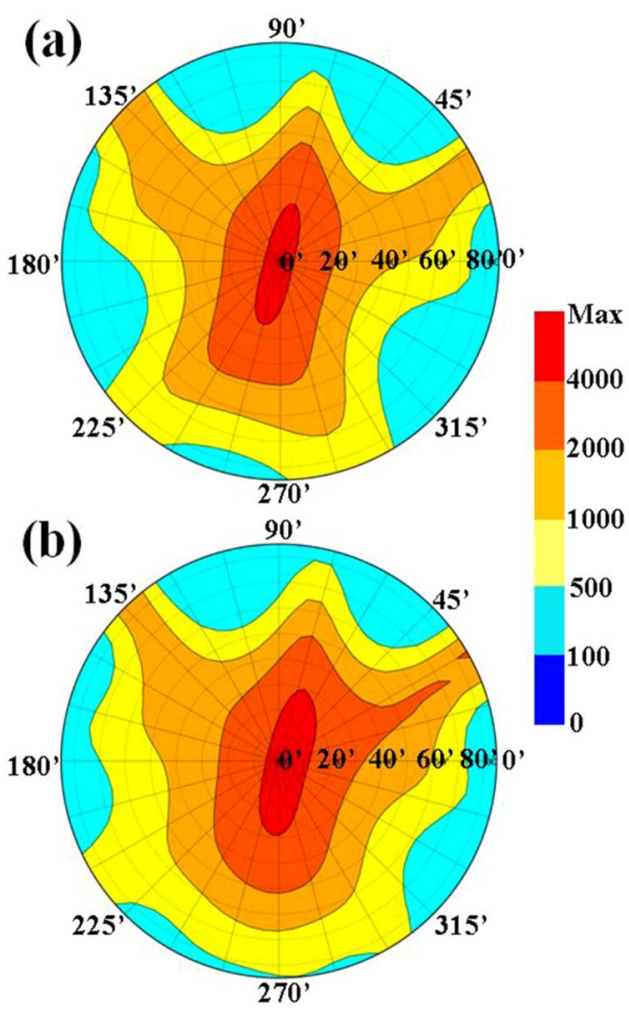

Fig. 5. Isocontrast contour plots of biaxial film-compensated (a) p-FFS and (b) p-PCFFS cells. $\lambda=550 \mathrm{~nm}$.

are often used [29], while a biaxial film is preferred for high-end LCDs. Hence, in our simulations, both FFS and PCFFS cells are compensated by adding a half-wave biaxial film before the analyzer in order to reduce the off-axis light leakage at dark state. The parameters of the biaxial film are as follows: $n_{x}=1.521$, $n_{y}=1.519, n_{z}=1.520$ and thickness is $137.5 \mu \mathrm{m}$. The $n_{x}$ axis of the biaxial film is perpendicular to the rubbing direction; that is, $100^{\circ}$ and $170^{\circ}$ w.r.t. the pixel electrode for LC cells employing negative and positive LC material, respectively.

Fig. 5(a) and (b) plots the simulated isocontrast contours of film-compensated p-FFS and p-PCFFS cells, respectively. We can see that both p-FFS and p-PCFFS possess an over $85^{\circ}$ viewing zone with contrast ratio greater than $100: 1$, while p-PCFFS shows slightly better viewing angle than p-FFS. Since their dark state is identical due to the same homogenous alignment, cell gap, and polarizers, the contrast ratio is mainly determined by the bright state. As explained above, the field generated by the patterned common electrodes in the p-PCFFS cell creates multi-domain LC distribution at the bright state, thus rending the viewing angle wider than that of p-FFS.

\section{Fabrication Tolerance}

Besides the aforementioned electro-optic properties, our PCFFS mode also possesses a large fabrication tolerance, as described in the following.

Firstly, the VT characteristics of the PCFFS mode are insensitive to the $\alpha$ angle although it has an optimal value to achieve fast response time. Fig. 6(a)-(d) compares the on-state transmittance profiles of p-PCFFS cells using different $\alpha$ angles with that of a conventional p-FFS cell, and the numerical values of 

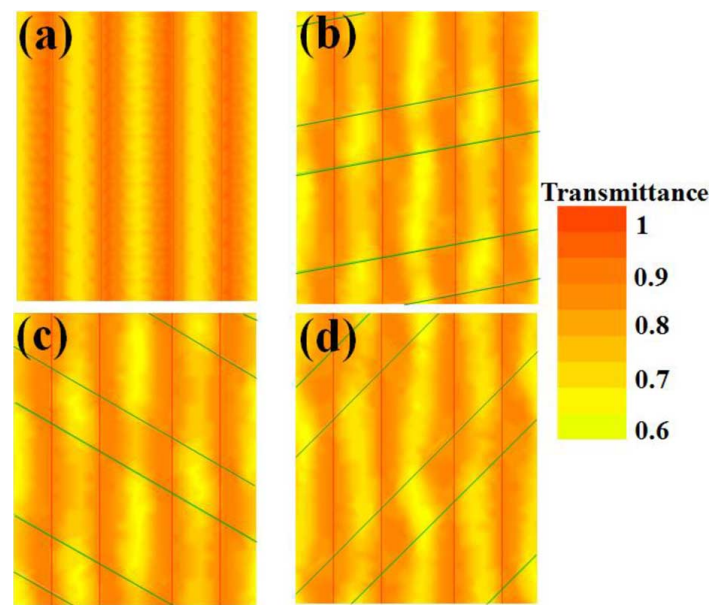

Fig. 6. On-state transmittance profiles of (a) p-FFS, and p-PCFFS modes with different $\alpha$ angles: (b) $\alpha=10^{\circ}$, (c) $\alpha=-30^{\circ}$ and (d) $\alpha=45^{\circ}(\lambda=$ $550 \mathrm{~nm})$.

TABLE II

Simulated Peak Transmittance and Operation Voltage of P-FFS AND P-PCFFS MODES WITH DiFFERENT $\alpha$ ANGLES $(\lambda=550 \mathrm{~nm})$

\begin{tabular}{|c|c|c|}
\hline LC Cell & $T_{\max }$ & $V_{\text {on }}(\mathrm{V})$ \\
\hline $\mathrm{p}-\mathrm{FFS}$ & $81.9 \%$ & 5.6 \\
\hline p-PCFFS $\alpha=10^{\circ}$ & $81.4 \%$ & 5.5 \\
\hline p-PCFFS $\alpha=-30^{\circ}$ & $81.7 \%$ & 5.5 \\
\hline p-PCFFS $\alpha=45^{\circ}$ & $81.2 \%$ & 5.5 \\
\hline
\end{tabular}

peak transmittance $\left(T_{\max }\right)$ and on-state voltage $\left(V_{\text {on }}\right)$ are listed in Table II. It is clearly shown that when $\alpha$ angle increases from $-30^{\circ}, 10^{\circ}$ to $45^{\circ}$, both $T_{\max }$ and $V_{\text {on }}$ remain nearly unchanged. The same trend applies to n-PCFFS as well. This insensitivity to $\alpha$ angle is very attractive, as it implies we have large tolerance in device fabrication because a small error in $\alpha$ angle would not influence the VT characteristics of PCFFS cells.

Secondly, the VT curves of PCFFS cells are also insensitive to the dimension of bottom common electrodes within a certain range. Fig. 7 shows the dependences of peak transmittance and on-state voltage on the $G_{2} / W_{2}$ ratio of bottom common electrodes for p-PCFFS. Both $T_{\max }$ and $V_{\text {on }}$ are insensitive to the $G_{2} / W_{2}$ ratio when $G_{2} / W_{2} \leq 1.0$. This feature offers freedom during device fabrication, as a small error in $G_{2} / W_{2}$ would not affect the electro-optic properties of p-PCFFS cells as long as it is kept below 1.0. Beyond this region, $T_{\max }$ decreases and $V_{\text {on }}$ increases dramatically. The same trend applies to n-PCFFS as well, but the tolerance range is even larger: $G_{2} / W_{2} \leq 1.5$, indicating that n-PCFFS is less sensitive to the $G_{2} / W_{2}$ ratio than p-PCFFS. This is because the positive LC directors tend to follow the electric field direction, which is determined by $G_{2} / W_{2}$ ratio. Hence, in order to achieve high transmittance and low operation voltage, $G_{2} / W_{2}$ ratio should not exceed 1.0 or 1.5 for $\mathrm{p}$ - and n-PCFFS cells, respectively.

In brief, the proposed PCFFS mode exhibits nearly identical VT characteristics to the conventional FFS mode. Meanwhile, their VT properties are insensitive to $\alpha$ angle and $G_{2} / W_{2}$ ratio, which provide large fabrication tolerance. More attractively, our PCFFS is able to achieve much faster response time than FFS, as will be outlined in Section IV.

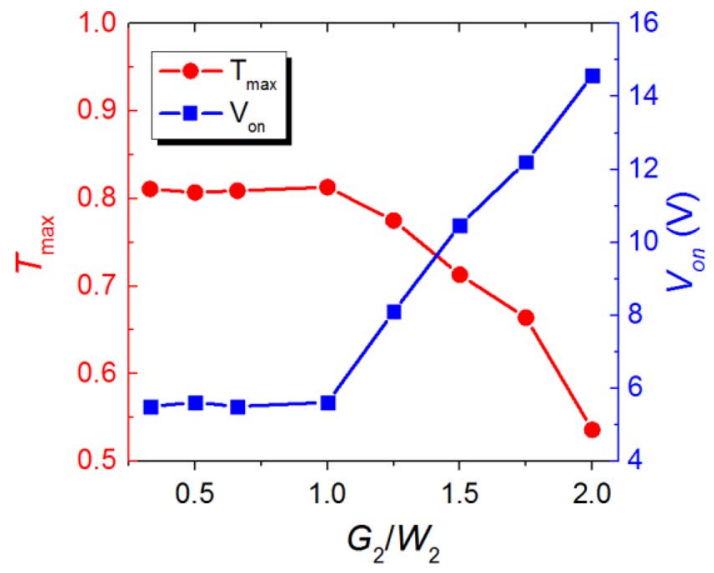

Fig. 7. Dependence of $\mathrm{T}_{\max }$ and $\mathrm{V}_{\text {on }}$ on the $G_{2} / W_{2}$ ratio for p-PCFFS.

\section{Simulation Results: Dynamic Response}

\section{A. Response Time}

During decay process, a restoring voltage pulse is applied between neighboring common electrodes while the top pixel electrodes are kept floated. Hence, the in-plane field generated by the restoring voltage would pull the LC directors back to their initial states. Note that the value of $\alpha$ angle is very crucial to the decay time. Take p-PCFFS as an example, the optimal $\alpha$ angle for achieving the fastest decay time is $10^{\circ}$. In the full-bright state, the LC directors are reoriented at $\sim 40^{\circ}-50^{\circ}$ from their initial alignment direction $\varphi$ [2]. Thus, the value of $\alpha$ angle should be optimized within the range $\varphi-90^{\circ}<\alpha<\varphi-45^{\circ}$ in order to maximize the restoring torque experienced by the LCs. In simulations, we set the rubbing angle of p-PCFFS at $\varphi=80^{\circ}$. Accordingly, $\alpha$ angle should be optimized between $-10^{\circ}$ and $35^{\circ}$.

Fig. 8(a) depicts the time-dependent transmittance (TT) curves of the p-PCFFS cells with $\alpha$ increasing from $-10^{\circ}, 10^{\circ}$ to $30^{\circ}$. Although the rise time is not emphasized in this study, our PCFFS cell still shows faster turn-on time than p-FFS cell (14.15 ms versus $20.62 \mathrm{~ms}$ ). During the relaxation process, a $15 \mathrm{~V}$ restoring voltage is applied to the common electrodes in the p-PCFFS cells. Although $\alpha$ angle does not influence the VT curves as discussed above, it determines the decay processes. By setting $\alpha$ at $10^{\circ}$, we are able to achieve the fastest decay time, which is $\sim 2.6 \times$ faster than that of conventional FFS mode ( $6.14 \mathrm{~ms}$ versus $16.22 \mathrm{~ms}$ ). Moreover, if a material with reduced viscosity is employed [12], the cell gap can be further reduced and response time will become even shorter for PCFFS modes.

Please note here the decay processes of PCFFS cells consist of two relaxation processes: the fast relaxation caused by the short restoring pulse is followed by a slow decay process originated from the spontaneous elastic relaxation. This is because only the in-plane horizontal electric field is effective in pulling the LC back to its original alignment direction. The effective restoring torque on the top of common electrodes is very small because the electric fields in these regions are mainly along the vertical direction, as shown in Fig. 8(b). Thus, by only applying the restoring voltage, the first faster decay process cannot reach 

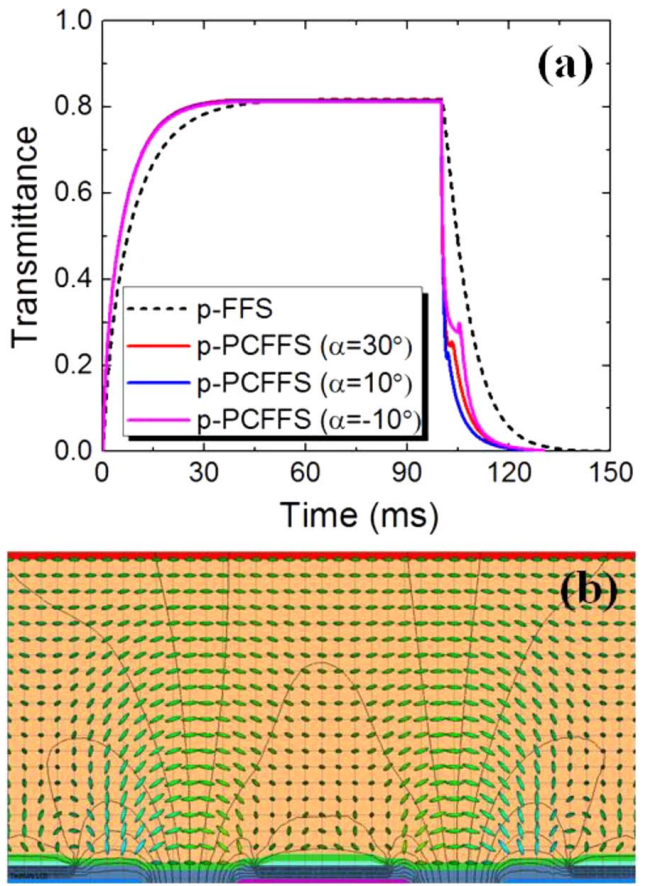

Fig. 8. (a) Time-dependent transmittance curves of p-FFS and p-PCFFS cells with different $\alpha$ angles and (b) electric potential profiles of p-PCFFS when a restoring voltage is applied.

dark state. If the transmittance of final level during a decay process is lower than the lowest transmittance the first decay process could reach, the second spontaneous relaxation process is required.

Similarly, by applying a restoring pulse, the decay time of n-PCFFS can be dramatically reduced as well. Different from $\mathrm{p}-$ PCFFS, $\alpha$ is set at $-20^{\circ}$ for n-PCFFS to achieve its fastest decay time, which is more than $2 \times$ faster than conventional n-FFS (7.43 $\mathrm{ms}$ versus $16.87 \mathrm{~ms}$ ). Thus, by applying the restoring pulse for a short period, the decay time of both p-PCFFS and n-PCFFS cells can be shortened.

\section{B. Passivation Layer Effect}

During the decay process, the restoring electric field is generated between top pixel and bottom common electrodes by penetrating through the passivation layer. Due to the dielectric properties of passivation layer [30], part of the applied voltage is shielded. Therefore, a high restoring voltage is required to accelerate the decay process. Fig. 9(a) and (b) compares the electric potential intensity profiles in two PCFFS cells with $0.40-\mu \mathrm{m}$ and $0.15-\mu \mathrm{m}$ passivation layers when a $15 \mathrm{~V}$ restoring voltage is applied. It clearly shows that the field penetrates deeper into the LC bulk in the cell with $0.15-\mu \mathrm{m}$ passivation whereas the voltage is more shielded in the other cell by the $0.40-\mu \mathrm{m}$ passivation layer. To better understand the difference, we investigated the change of electric field intensity at position F [as defined in Fig. 4(a)], where the field is mainly along in-plane horizontal direction. The distribution of electric field is plotted in Fig. 9(c). Because more voltage is shielded by the passivation layer, the penetration depth of the in-plane field is much shallower in the $0.40-\mu \mathrm{m}$ passivation cell than that in the other one. Therefore, a thinner passivation is preferred to reduce the restoring voltage. If we
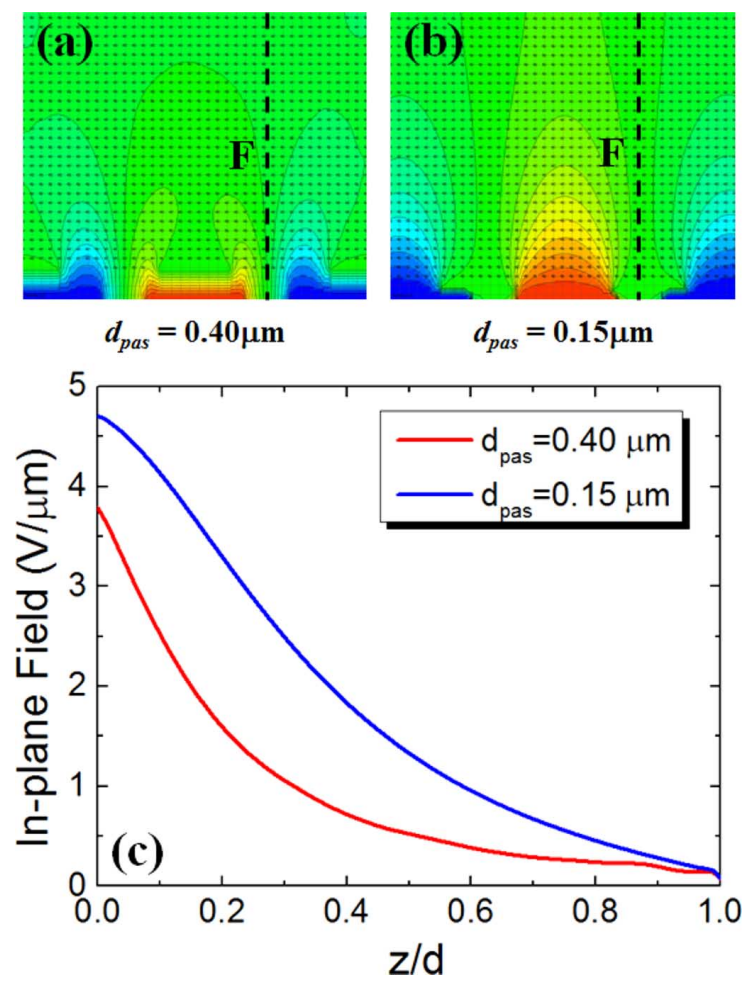

Fig. 9. (a), (b) Electric potential profiles in PCFFS cells with different passivation layer thickness when a restoring voltage of $15 \mathrm{~V}$ is applied; and (c) the distribution of electric field in the cell at position F in Fig. 4(a).

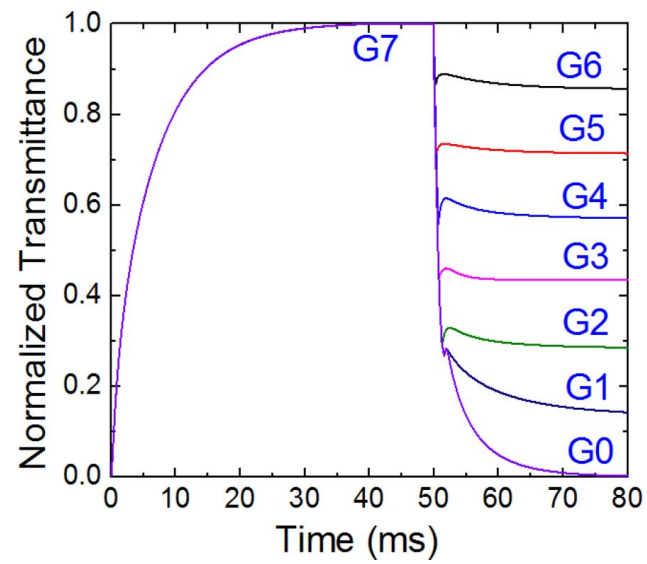

Fig. 10. Time-dependent transmittance curves of the decay processes from G7 to lower gray levels and their response times.

can reduce the passivation thickness to $0.10 \mu \mathrm{m}$, the required restoring voltage would be further reduced to $10 \mathrm{~V}$.

\section{GTG Decay Time}

To evaluate the GTG decay time, we divided the VT curve uniformly into 8 gray levels (G0-G7). As usual, the decay time is defined as $90 \%-10 \%$ transmittance change. The restoring pulse is applied to bottom common electrodes for a short period until the transmittance decays to the designated gray level. Then a bias voltage is applied to hold the transmittance at the targeted final gray level. Details of this driving method have been reported in [16], [31], and [32].

The transient transmittance decay processes from G7 to lower gray levels of the p-PCFFS cell are shown in Fig. 10. The decay 
TABLE III

Calculated GTG Decay Time of p-PCFFs at $\mathrm{T}=23^{\circ} \mathrm{C}$ AND $\lambda=550 \mathrm{~nm}$ (UNIT: $\mathrm{ms})$

\begin{tabular}{|c|c|c|c|c|c|c|c|c|}
\hline Level & $\mathbf{0}$ & $\mathbf{1}$ & $\mathbf{2}$ & $\mathbf{3}$ & $\mathbf{4}$ & $\mathbf{5}$ & $\mathbf{6}$ & $\mathbf{7}$ \\
\hline $\mathbf{0}$ & & & & & & & & \\
\hline $\mathbf{1}$ & 5.48 & & & & & & & \\
\hline $\mathbf{2}$ & 5.62 & 4.88 & & & & & & \\
\hline $\mathbf{3}$ & 6.04 & 4.98 & 0.47 & & & & & \\
\hline $\mathbf{4}$ & 6.04 & 5.02 & 0.51 & 0.33 & & & & \\
\hline $\mathbf{5}$ & 6.10 & 5.04 & 0.62 & 0.47 & 0.29 & & & \\
\hline $\mathbf{6}$ & 6.08 & 5.04 & 0.73 & 0.59 & 0.43 & 0.24 & & \\
\hline $\mathbf{7}$ & 6.14 & 5.08 & 0.88 & 0.77 & 0.54 & 0.39 & 0.31 & \\
\hline
\end{tabular}

TABLE IV

Calculated GTG Decay Time of P-FFS at T $=23^{\circ} \mathrm{C}$ AND $\lambda=550 \mathrm{~nm}$ (UNIT: ms)

\begin{tabular}{|c|c|c|c|c|c|c|c|c|}
\hline Level & $\mathbf{0}$ & $\mathbf{1}$ & $\mathbf{2}$ & $\mathbf{3}$ & $\mathbf{4}$ & $\mathbf{5}$ & $\mathbf{6}$ & $\mathbf{7}$ \\
\hline $\mathbf{0}$ & & & & & & & & \\
\hline $\mathbf{1}$ & 13.79 & & & & & & & \\
\hline $\mathbf{2}$ & 14.02 & 27.82 & & & & & & \\
\hline $\mathbf{3}$ & 14.19 & 26.56 & 26.44 & & & & & \\
\hline $\mathbf{4}$ & 14.71 & 25.66 & 25.73 & 25.06 & & & & \\
\hline $\mathbf{5}$ & 15.03 & 25.27 & 25.53 & 25.78 & 23.73 & & & \\
\hline $\mathbf{6}$ & 15.37 & 25.21 & 25.54 & 25.07 & 24.24 & 23.41 & & \\
\hline $\mathbf{7}$ & 16.22 & 25.58 & 26.11 & 25.90 & 25.32 & 25.43 & 24.45 & \\
\hline
\end{tabular}

TABLE V

Calculated GTG Decay Time of N-PCFFS at $\mathrm{T}=23^{\circ} \mathrm{C}$ AND $\lambda=550 \mathrm{~nm}$ (UNIT: ms)

\begin{tabular}{|c|c|c|c|c|c|c|c|c|}
\hline Level & $\mathbf{0}$ & $\mathbf{1}$ & $\mathbf{2}$ & $\mathbf{3}$ & $\mathbf{4}$ & $\mathbf{5}$ & $\mathbf{6}$ & $\mathbf{7}$ \\
\hline $\mathbf{0}$ & & & & & & & & \\
\hline $\mathbf{1}$ & 5.76 & & & & & & & \\
\hline $\mathbf{2}$ & 6.17 & 4.87 & & & & & & \\
\hline $\mathbf{3}$ & 6.58 & 5.33 & 0.32 & & & & & \\
\hline $\mathbf{4}$ & 6.81 & 5.61 & 0.53 & 0.37 & & & & \\
\hline $\mathbf{5}$ & 7.09 & 5.83 & 0.92 & 0.64 & 0.29 & & & \\
\hline $\mathbf{6}$ & 7.15 & 5.97 & 1.11 & 0.75 & 0.53 & 0.31 & & \\
\hline $\mathbf{7}$ & 7.43 & 6.23 & 1.39 & 0.84 & 0.63 & 0.37 & 0.25 & \\
\hline
\end{tabular}

time of the first relaxation process expedited by the electric field is very fast, while the decay processes to lower gray levels (G0 and G1) require a longer time for the elastic relaxation of LC directors to accomplish. The calculated GTG decay time of p-PCFFS is summarized in Table III. Most GTG decay times are below $1 \mathrm{~ms}$ except those whose final level is G0 or G1, and the average GTG decay time is $2.82 \mathrm{~ms}$ at $23^{\circ} \mathrm{C}$. Compared to the conventional p-FFS mode, whose average GTG response time is $22.75 \mathrm{~ms}$ as listed in Table IV, our PCFFS cell shows $>8 \mathrm{X}$ faster GTG decay time. Furthermore, the decay time will be reduced if LC with a lower viscosity [12] is employed or a higher restoring voltage is applied.

The GTG decay time of n-PCFFS and n-FFS modes are also calculated, as listed in Tables V and VI, respectively. Compared to $\mathrm{n}$-FFS, the proposed n-PCFFS is able to achieve $>7 \times$ faster GTG decay time ( $3.21 \mathrm{~ms}$ versus $24.05 \mathrm{~ms})$ at $23^{\circ} \mathrm{C}$. This GTG decay time is even faster than that of MVA LCD, whose GTG response time is $\sim 5 \mathrm{~ms}$. Hence, this fast-switching mode has promising applications for eliminating motion image blurs.

\section{CONCLUSION}

We proposed a new PCFFS mode to achieve fast decay time. Compared to conventional FFS mode, the new PCFFS exhibits
TABLE VI

Calculated GTG Decay Time of N-FFS at $\mathrm{T}=23^{\circ} \mathrm{C}$ AND $\lambda=550 \mathrm{~nm}$ (UNIT: $\mathrm{ms})$

\begin{tabular}{|c|c|c|c|c|c|c|c|c|}
\hline Level & $\mathbf{0}$ & $\mathbf{1}$ & $\mathbf{2}$ & $\mathbf{3}$ & $\mathbf{4}$ & $\mathbf{5}$ & $\mathbf{6}$ & $\mathbf{7}$ \\
\hline $\mathbf{0}$ & & & & & & & & \\
\hline $\mathbf{1}$ & 13.77 & & & & & & & \\
\hline $\mathbf{2}$ & 13.97 & 24.68 & & & & & & \\
\hline $\mathbf{3}$ & 14.25 & 30.37 & 20.22 & & & & & \\
\hline $\mathbf{4}$ & 14.73 & 29.35 & 29.39 & 27.57 & & & & \\
\hline $\mathbf{5}$ & 15.07 & 28.79 & 29.05 & 27.37 & 25.02 & & & \\
\hline $\mathbf{6}$ & 16.81 & 28.74 & 28.91 & 27.54 & 25.34 & 22.93 & & \\
\hline $\mathbf{7}$ & 16.87 & 29.31 & 29.77 & 28.78 & 26.97 & 24.97 & 22.84 & \\
\hline
\end{tabular}

nearly identical VT characteristics without tradeoffs in electrooptic performance. Besides, the PCFFS possess a large fabrication tolerance, which is a very favorable feature. By applying a restoring voltage pulse on the neighboring common electrodes, the LC directors are expedited to relax back to their initial alignment direction. With proper cell configuration and driving schemes, we are able to reduce the average GTG decay time by $>8 \times$ for $\mathrm{p}$-PCFFS and $>7 \times$ for n-PCFFS; as compared to FFS. This fast-response mode has potential applications for reducing motion picture image blurs.

\section{REFERENCES}

[1] S. H. Lee, S. L. Lee, and H. Y. Kim, "Electro-optic characteristics and switching principle of a nematic liquid crystal cell controlled by fringefield switching," Appl. Phys. Lett., vol. 73, pp. 2881-2883, 1998.

[2] Y. Chen, Z. Y. Luo, F. L. Peng, and S. T. Wu, "Fringe-field switching with a negative dielectric anisotropy liquid crystal," $J$. Display Technol., vol. 9, no. 2, pp. 74-77, Feb. 2013.

[3] F. Yang, J. Zhang, X. Xiang, Y. Ko, and F. Bai, "High performance advanced super dimension switch (ADS) mode LCD with negative dielectric anisotropy LC optimization," in SID Int. Symp. Dig. Tech. Papers, 2014, vol. 45, pp. 318-321.

[4] D. H. Kim, Y. J. Lim, D. E. Kim, H. Ren, S. H. Ahn, and S. H. Lee, "Past, present, and future of fringe-field switching-liquid crystal display," Liq. Cryst., vol. 15, pp. 99-106, 2014.

[5] T. Tsuruma, Y. Goto, A. Higashi, M. Watanabe, H. Yamaguchi, and T. Tomooka, "Novel image sticking model in the fringe field switching mode based on the flexoelectric effect," in Proc. Eurodisplay, 2011, vol. 11, pp. 13-16.

[6] D. Xu et al., "Image sticking in liquid crystal displays with lateral electric fields," J. Appl. Phys., vol. 116, 2014, Art. ID 193102.

[7] L. Lu, A. K. Bhowmik, and P. J. Bos, "The effect of dielectric constant on ion adsorption in liquid crystal devices," Liq. Cryst., vol. 40, pp. 7-13, 2013.

[8] R. Hatsumi et al., "FFS-mode OS-LCD for reducing eye strain," J. Soc. Inf. Display, vol. 21, pp. 442-450, 2013.

[9] S. D. Brotherton, Introduction to Thin Film Transistors: Physics and Technology of TFTs. New York, NY, USA: Springer, 2013.

[10] T. Kurita, "Moving picture quality improvement for hold-type AM-LCDs," in SID Int. Symp. Dig. Tech. Pap., 2012, vol. 32, pp. 986-989.

[11] E. Lueder, Liquid Crystal Displays: Addressing Schemes and ElectroOptical Effects. Chichester, U.K.: Wiley, 2001.

[12] H. Chen et al., "High performance liquid crystal displays with a low dielectric constant material," Opt. Mater. Exp., vol. 4, pp. 2262-2273, 2014.

[13] A. Takeda et al., "A super-high image quality multi-domain vertical alignment LCD by new rubbing-less technology," in SID Int. Symp. Dig. Tech. Papers, 1998, vol. 29, pp. 1077-1100.

[14] Y. Tanaka, Y. Taniguchi, T. Sasaki, A. Takeda, Y. Koibe, and K. Okamoto, "A new design to improve performance and simplify the manufacturing process of high-quality MVA TFT-LCD panels," in SID Int. Symp. Dig. Tech. Papers, 1999, vol. 30, pp. 206-209.

[15] C. Y. Xiang, X. W. Sun, and X. J. Yin, "Fast response wide viewing angle liquid crystal cell with double-side fringe-field switching," Appl. Phys. Lett., vol. 83, pp. 5154-5156, 2003. 
[16] Y. Li, Z. Ge, R. Lu, M. Jiao, and S. T. Wu, "Fast-response liquid-crystal displays using crossed fringe fields," J. Soc. Inf. Display, vol. 16, pp. 1069-1074, 2008

[17] T. H. Choi, J. W. Kim, and T. H. Yoon, "Fast in-plane switching of negative liquid crystals using crossed patterned electrodes," Jpn. J. Appl. Phys., vol. 53, p. 081701, 2014.

[18] Y. Kim et al., "Fast response time of fringe-field switching liquid crystal mode devices with reactive mesogens in a planar alignment layer," J. Phys. D: Appl. Phys., vol. 46, 2013, Art. ID 485306.

[19] Y. J. Lim, Y. E. Choi, J. H. Lee, G. D. Lee, L. Komitov, and S. H. Lee, "Effects of three-dimensional polymer networks in vertical alignment liquid crystal display controlled by in-plane field," Opt. Exp., vol. 22 , pp. 10634-10641, 2014.

[20] J. I. Baek et al., "Fast in-plane switching of a liquid crystal cell triggered by a vertical electric field," Jpn. J. Appl. Phys., vol. 48, p. 104505, 2009.

[21] D. Xu, L. Rao, C. D. Tu, and S. T. Wu, "Nematic liquid crystal display with submillisecond grayscale response time," J. Display Technol., vol. 9, no. 1, pp. 67-70, Jan. 2013.

[22] C. Y. Xiang, X. W. Sun, and X. J. Yin, "The electro-optic properties of a vertically aligned fast response liquid crystal display with threeelectrode driving," J. Phys. D: Appl. Phys., vol. 37, pp. 994-997, 2004.

[23] M. K. Park et al., "Mixed-field-switching liquid crystal mode using in-plane and fringe fields self-adjusted by bottom floating electrode for transmittance enhancement," Liq. Cryst., vol. 41, pp. 908-919, 2014.

[24] D. J. Channin, "Triode optical gate: A new liquid crystal electro-optic device," Appl. Phys. Lett., vol. 11, pp. 603-605, 1975.

[25] C. Y. Xiang, J. X. Guo, X. W. Sun, X. J. Yin, and G. J. Qi, “A fast response, three-electrode liquid crystal device," Jpn. J. Appl. Phys., vol. 42, pp. L763-L765, 2003.

[26] J. W. Kim, T. H. Choi, and T. H. Yoon, "Fast switching of vertically aligned negative liquid crystals by optically hidden relaxation," Liq. Cryst., vol. 41, pp. 1212-1217, 2014.

[27] K. H. Kim, B. H. Yu, S. W. Choi, S. W. Oh, and T. H. Yoon, "Dual mode switching of cholesteric liquid crystal device with three-terminal electrode structure," Opt. Express, vol. 20, pp. 24376-24381, 2012.

[28] Y. Chen, F. Peng, T. Yamaguchi, X. Song, and S. T. Wu, "High performance negative dielectric anisotropy liquid crystals for display applications," Crystals, vol. 3, pp. 483-503, 2013.

[29] Z. Luo, D. Xu, and S. T. Wu, "Emerging quantum-dots-enhanced LCDs," J. Display Technol., vol. 10, no. 3, pp. 526-539, Mar. 2014.

[30] M. Jiao, Z. Ge, Q. Song, and S. T. Wu, "Alignment layer effects on thin liquid crystal cells," Appl. Phys. Lett., vol. 92, 2008, Art. ID 061102.

[31] J. W. Kim, T. H. Choi, and T. H. Yoon, "Drive schemes for sub-millisecond switching of LCD panels," in SID Int. Symp. Dig. Tech. Pap., 2014, vol. 45 , pp. $1460-1462$.

[32] J. W. Kim, S. T. Shin, and T. Y. Yoon, "Technologies for sub-millisecond response time of nematic liquid crystals," Mol. Cryst. Liq. Cryst., vol. 595, pp. 1-8, 2014.

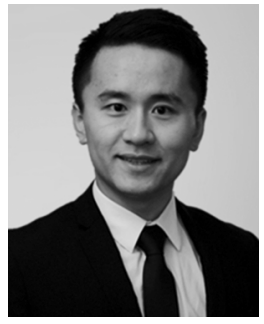

Daming Xu received the B.S. degree in information engineering from Southeast University, Nanjing, China, in 2011, and is currently working toward the $\mathrm{Ph} . \mathrm{D}$. degree at the College of Optics and Photonics, University of Central Florida, Orlando, FL, USA.

His current research focuses on fast-response nematic liquid crystal devices and blue phase liquid crystal displays. He has published 17 journal papers and 14 conference papers.

Mr. Xu was the recipient of SID Distinguished Student Paper Award and SPIE Research Excellence Award. He was President of SID student chapter at University of Central Florida in 2014

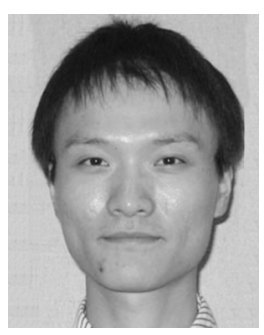

Haiwei Chen received the B.S. degree in optoelectronic information engineering from Beihang University, Beijing, China, in 2013, and is currently working toward the Ph.D. degree at the College of Optics and Photonics, University of Central Florida, Orlando, FL, USA.

His current research focuses on liquid crystal devices design and material development.

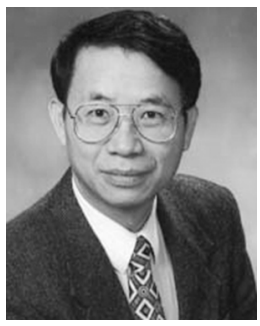

Shin-Tson Wu (M'98-SM'99-F'04) received the B.S. degree in physics from National Taiwan University, Taipei, Taiwan, and the Ph.D. degree from the University of Southern California, Los Angeles, CA, USA.

$\mathrm{He}$ is a Pegasus Professor with the College of Optics and Photonics, University of Central Florida, Orlando, Orlando, FL, USA

Dr. Wu is a Fellow of the Society of Information Display (SID), the Optical Society of America (OSA), and SPIE. He is among the first six inductees of the Florida Inventors Hall of Fame (2014) and is a Charter Fellow of the National Academy of Inventors (2012). He was the recipient of the OSA Esther Hoffman Beller Medal (2014), SID Slottow-Owaki prize (2011), OSA Joseph Fraunhofer award (2010), SPIE G. G. Stokes award (2008), and SID Jan Rajchman prize (2008). He was the founding Editor-in-Chief of IEEE/OSA JOURNAL OF DISPLAY TECHNOLOGY.

Ming-Chun Li, photograph and biography not available at the time of publication.

Seok-Lyul Lee, photograph and biography not available at the time of publication.

Wen-Ching Tsai, photograph and biography not available at the time of publication. 